\title{
THE INFLUENCE OF CULTIVATION SYSTEM ON BIOCHEMICAL CONTENT OF STRAWBERRY FRUITS
}

\author{
Valda LAUGALE ${ }^{1 *}$, Sarmite STRAUTINA ${ }^{2}$, Inta KRASNOVA ${ }^{2}$, Dalija SEGLINA ${ }^{2}$, \\ Kaspars KAMPUSS ${ }^{3}$ \\ ${ }^{1}$ Pūre Horticultural Research Centre, Abavas 2, Pūre, Tukuma nov., LV-3124, Latvia \\ ${ }^{2}$ Latvia State Institute of Fruit-Growing, Graudu 1, Dobele, LV-3701, Latvia \\ ${ }^{3}$ Latvia University of Agriculture, Liela iela 2, Jelgava, LV-3001, Latvia \\ Received: April 3, 2014; Accepted: November 4, 2014
}

\begin{abstract}
Three cultivars 'Zefyr', 'Honoeoye' and 'Polka' were grown on beds mulched with black plastic or without it, as well as in low tunnels covered by transparent polyethylene film or Agronet (Pegas agro, $17 \mathrm{~g} \cdot \mathrm{m}^{-2}$ ), or without covering. The quality of fruits determined by content of ascorbic acid, titratable acidity, soluble solids, total content of phenolics and anthocyanins, antiradical activity, and radical scavenging activity (RSA) was evaluated during two seasons. Significant differences in phytochemical contents within strawberry cultivars, production seasons and cultivation methods were stated. Cultivar had the greatest influence on the content of ascorbic acid, total phenolics and RSA among all the studied factors. The amount of anthocyanins was mainly influenced by plant covering, while the annual climatic conditions had the greatest impact on the amount of titratable acidity and soluble solids. Soil mulching had the lowest influence on the chemical content of fruits.
\end{abstract}

Key words: Fragaria $\times$ ananassa Duch., plant covering, mulch, cultivar, season

\section{INTRODUCTION}

Strawberry is one of the most popular berry crops in Latvia. Production season for local strawberry is quite short and mostly lasts from mid-June to mid-July. It causes a sharp decrease of fruit price during the peak of harvesting and difficulties in marketing. By this reason, there is an interest in the extending of strawberry cropping period, especially through earlier fruiting when prices are highest. This result could be obtained using microtunnels (Medina 2008; Martinsson 1997) or soil mulching (Himelrick 1996; Kikas 1997).

Strawberry is a source of many bioactive phytochemicals as ascorbic acid, polyphenolic compounds and ellagic acid. Previous studies show that strawberries have more antioxidants than apple, apricot, peaches or kiwifruit (Scalzo et al. 2004). Antioxidants are considered to help in preventing of several diseases like cancer and cardiovascular diseases (Hannum 2004; Manach et al. 2004; Weaver et al. 2009; Jaroslawska et al. 2011). According to Giampieri et al. (2012), content of micronutrients and phytochemicals may greatly vary depending on cultivar and cultivation practices (Giampieri et al. 2012).

The field experiment was undertaken to evaluate fruit quality of strawberry from different cultivation systems favouring earlier fruit ripening in Latvia conditions.

\section{MATERIALS AND METHODS}

The field experiment was located in Pūre, Tukums region, Latvia, at $57^{\circ} 02^{\prime}$ northern latitude and $22^{\circ} 52^{\prime}$ eastern longitude. Freshly dug plants cvs Zefyr', 'Honoeoye' and 'Polka' were planted in the loamy and sod calcareous soil that lays on dolomite rock in September of 2007. Two-rows of low beds, $0.3 \mathrm{~m}$ between rows and $0.4 \mathrm{~m}$ between plants, and $1.5 \mathrm{~m}$ between bed centres were designed. Plants were watered and fertigated by drip irrigation. 
Weeding on beds was performed manually and herbicide Basta ${ }^{\circledR}$ (glufosinate-ammonium $200 \mathrm{~g} \cdot \mathrm{L}^{-1}$ ) was used in rows between beds. Split-split-plot experimental design with three factors was used in the trial: 1 - soil mulching with black plastic or without mulching, 2 - plant covering with transparent polyethylene film (120 $\mu \mathrm{m}$ thick) or Agronet (Pegas agro, $17 \mathrm{~g} \cdot \mathrm{m}^{-2}$ ) using low tunnels or without covering, 3 - cultivars. Each plot of $10 \mathrm{~m}^{2}$ was replicated four times. The tunnels of $0.5 \mathrm{~m}$ high and $1 \mathrm{~m}$ wide were applied every year at the beginning of April. Covers were opened at day-time during flowering period and when temperature exceeded $25^{\circ} \mathrm{C}$. They were removed fully at the beginning of harvesting.

The following chemical fruit compounds were determined in years 2008 and 2009: the content of ascorbic acid by the iodine method (Moor et al. 2005), the titratable acidity by titrating with $0.1 \mathrm{~N}$ $\mathrm{NaOH}$ (ISO 750:1998); the amount of soluble solids by hand refractometer (ISO 2173: 2003), the total content of phenolics by the spectrophotometric Folin-Ciocalteau method using a spectrometer UV1650-PC at wave length $765 \mathrm{~nm}$ (Singleton et al. 1999), the total content of anthocyanins by the conventional method using a spectrometer UV-1650PC at wavelength $535 \mathrm{~nm}$ (Moor et al. 2005), the antiradical activity by 2.2-diphenyl-1-picrylhydrazyl (DPPH) method (Miliauskas et al. 2004) and radical scavenging activity (RSA) in percentage was calculated (Seglina et al. 2008).

The contents of ascorbic acid, titratable acidity, phenolics and anthocyanins, and antiradical activity were determined at the Biochemical Laboratory of Latvia State Institute of Fruit Growing and the content of soluble solids at the Laboratory of Pūre HRC. The fruits for analyses were harvested from each treatment in the time of maximum colouring. The samples were analysed on the day of harvest. The size of sample was $1 \mathrm{~kg}$ of ripe fruits from every treatment.

Data were statistically processed by using MS Excel and STATISTICA ${ }^{\mathrm{TM}}$. Multifactorial analysis of variance and correlation were used. The significant differences between means were determined at $p=0.05$ by Duncan's multiple range test. The effect size of different factors was evaluated and $\eta^{2} \%$ calculated.

\section{RESULTS AND DISCUSSION}

The biochemical contents significantly varied within strawberry cultivars, cultural methods and years of the trial. The significant interactions between different factors were observed.

\section{Influence of season}

Years of study differed in weather conditions. The air temperature in April was higher by $0.3{ }^{\circ} \mathrm{C}$ and in May by $0.6^{\circ} \mathrm{C}$ in 2009 as compared to 2008 , but June was warmer in 2008. It influenced the beginning of cropping, but in different way in different treatments. Generally, the higher contents of total phenolics, anthocyanins and ascorbic acid were recorded in 2009 in comparison to 2008 (Table 1, 2), while a higher content of titratable acidity, soluble solids and RSA was detected in 2008 (Table 1, 2). A weather conditions in combinations with particular treatments affected mostly contents of titratable acidity and soluble solids (Table 3 ). Similar results were reported by Del Pozo-Insfran et al. (2006) and Diamanti et al. (2009).

Also, term of fruit sampling, which depends on weather conditions before fruit ripening is important for accumulation of analysed compounds (Krüger et al. 2009). The environment and the course of harvesting had a stronger influence on the fruit biochemical contents then a cultivation technique (Josuttis et al. 2009). In our investigation, the significant positive correlations between average air temperature in the decade before samples collection and content of total phenolics $(\mathrm{r}=0.36 ; \mathrm{p}<0.05 ; \mathrm{n}=36)$ and RSA $(\mathrm{r}=0.37$; $p<0.05 ; n=36$ ) were found during two years of investigation. Moreover, the significant negative correlations between sum of precipitations in the decade before fruit sampling and content of total phenolics $(\mathrm{r}=-0.38 ; \mathrm{p}<0.05 ; \mathrm{n}=36)$ and RSA $(\mathrm{r}=-0.43 ; \mathrm{p}<0.05 ; \mathrm{n}=36)$ were found. The influence of harvesting time on strawberry biochemical content was observed also by Mratinić and Milojević (2009). The great impact of environment on chemical properties and antioxidant contents in strawberry was also reported by Hansawasdi et al. (2006), Rutkowski et al. (2006), Seglina et al. (2008), Tulipani et al. (2009). 


\section{Influence of mulching}

The use of black plastic mulching significantly increased the yield and fruit size of strawberry (Laugale et al. 2012). However, it had a lesser impact on the strawberry biochemical contents than the other studied factors (Table 3 ). In both years of experiment, higher content of total phenolics, anthocyanins and titratable acidity was determined in fruits grown on bare ground comparing with black plastic mulching (Tables 1,2), while higher RSA was found in fruits grown in soil covered with black plastic. It agrees with observations of Josuttis et al. (2009), where the fruits from the matted row cultivation with polyethylene foil contained higher antioxidant capacity than those grown on bare ground. The amount of ascorbic acid did not differ significantly in the result of mulching in 2008, while in 2009 it was significantly lower in fruits from the treatments with mulching. The amount of soluble solids did not differ significantly dependent on mulching in 2009, while in 2008 it was significantly higher in fruits from this treatment.

\section{Influence of plants-covering with microtunnels}

Covering of plants accelerated the beginning of fruiting and increased strawberry productivity (Laugale et al. 2012). However, it affected fruits development resulting in smaller size of fruits in the treatments with polyethylene cover. As plant covers were removed at the beginning of fruit harvesting, they had no direct influence on chemical content at fruit ripening. Fruit chemical contents from covering treatments differed between years (Tables 1,2). The highest negative impact of plants-covering was stated for anthocyanin content (Table 3). In both years, the highest content of anthocyanins was determined in fruits from plants grown without covering (Tables 1,2). This result can be explained by higher air temperature and lower light transmittance during growth. Solar radiation and air temperature were mentioned as important factors influencing anthocyanin development (Del Pozo-Insfran et al. 2006). The negative influence of high temperature on development of anthocyanins in strawberry fruits was reported also by Ikeda et al. (2009).
Use of polyethylene covering on plants reduced significantly the content of titratable acidity in fruits in comparison to uncovered in year 2009. Other biochemical characteristics significantly varied between treatments and years.

\section{Influence of cultivar}

Genetic factors play an important role in berry fruit composition (Wang 2007). In our trial, cultivar had greater impact on content of total phenolics, ascorbic acid and RSA than mulching or covering (Table 3 ). Cultivars also varied in earliness, productivity and fruit size (Laugale et al. 2012). In both years of experiment, 'Zefyr' was characterised by the highest content of total phenolics, vitamin $\mathrm{C}$ and highest RSA activity than the other cultivars (Tables 1,2). It had the highest early yield, but also softer fruits than other cultivars (Laugale et al. 2012). In the above experiment, 'Honeoye' had the highest content of anthocyanins, while 'Polka' was more rich in soluble solids. 'Polka' was the most productive cultivar, but it had the lowest average fruit weight and the latest ripening time (Laugale et al. 2012).

Significant interaction between cultivars and cultivation systems was found, but results were dependent on the year of study. In 2008, the highest content of total phenolics in fruits of early ripening cultivars 'Zefyr' and 'Honeoye' was observed in the treatments where plants were grown on bare ground and under polyethylene covering (Table 1). The medium early cv. 'Polka' accumulated most of total phenolics in the fruits from the cultivation system, where black plastic mulch and Agronet covering were used. In 2009, the highest content of total phenolics for all cultivars was observed in fruits from plants grown on bare ground without plant covering in the spring (Table 2).

In both years of the study, the highest content of anthocyanins in fruits of 'Honeoye' and 'Polka', which have darker fruits colour, was found in the treatments without soil mulching or plants covering (Tables 1,2), whereas for 'Zefyr', cultivar of lighter fruits results differed between seasons and cultivation systems. 
Table 1. Biochemical content and radical scavenging activity in strawberry fruits in 2008 ( \pm standard deviation)

\begin{tabular}{|c|c|c|c|c|c|c|c|c|c|c|c|c|c|}
\hline \multicolumn{2}{|c|}{ Treatment* } & \multicolumn{2}{|c|}{$\begin{array}{l}\text { Phenolics } \\
\mathrm{mg} \cdot 100 \mathrm{~g}^{-1}\end{array}$} & \multicolumn{2}{|c|}{$\begin{array}{c}\mathrm{AN} * * \\
\mathrm{mg} \cdot 100 \mathrm{~g}^{-1}\end{array}$} & \multicolumn{2}{|c|}{$\begin{array}{c}\text { Ascorbic acid } \\
\mathrm{mg} \cdot 100 \mathrm{~g}^{-1}\end{array}$} & \multicolumn{2}{|c|}{$\begin{array}{l}\text { Titratable acid- } \\
\text { ity } \% \\
\end{array}$} & \multicolumn{2}{|c|}{$\begin{array}{c}\text { Soluble solids } \\
\text { OBrix }\end{array}$} & \multicolumn{2}{|c|}{$\mathrm{RSA} * * \%$} \\
\hline \multicolumn{14}{|c|}{ Without soil mulch } \\
\hline \multirow[t]{3}{*}{$\mathrm{N}$} & Zefyr & 248 & \pm 5 & 17.5 & \pm 0.6 & 79.4 & \pm 3.2 & 1.13 & \pm 0.04 & 10.6 & \pm 0.8 & 84.9 & \pm 0.8 \\
\hline & Honeoye & 209 & \pm 6 & 23.4 & \pm 0.3 & 67.0 & \pm 3.3 & 1.17 & \pm 0.02 & 10.1 & \pm 1.9 & 79.2 & \pm 0.7 \\
\hline & Polka & 162 & \pm 5 & 19.5 & \pm 0.3 & 64.2 & \pm 3.1 & 0.92 & \pm 0.06 & 11.9 & \pm 0.7 & 75.8 & \pm 0.6 \\
\hline \multirow[t]{3}{*}{$\mathrm{P}$} & Zefyr & 264 & \pm 6 & 16.3 & \pm 0.5 & 86.0 & \pm 2.2 & 1.01 & \pm 0.05 & 11.8 & \pm 0.5 & 86.2 & \pm 0.5 \\
\hline & Honeoye & 240 & \pm 6 & 17.8 & \pm 0.2 & 50.5 & \pm 1.2 & 1.01 & \pm 0.05 & 9.4 & \pm 0.7 & 82.9 & \pm 1.2 \\
\hline & Polka & 193 & \pm 7 & 14.3 & \pm 1.0 & 46.9 & \pm 5.5 & 0.95 & \pm 0.03 & 11.1 & \pm 0.9 & 82.1 & \pm 0.7 \\
\hline \multirow[t]{3}{*}{ A } & Zefyr & 226 & \pm 6 & 20.7 & \pm 1.4 & 68.4 & \pm 2.8 & 1.02 & \pm 0.03 & 11.4 & \pm 1.0 & 69.4 & \pm 0.3 \\
\hline & Honeoye & 214 & \pm 4 & 17.8 & \pm 2.0 & 72.0 & \pm 4.0 & 1.16 & \pm 0.03 & 8.8 & \pm 0.2 & 61.6 & \pm 1.0 \\
\hline & Polka & 156 & \pm 3 & 18.4 & \pm 0.1 & 65.1 & \pm 1.1 & 0.90 & \pm 0.03 & 11.0 & \pm 0.6 & 62.6 & \pm 2.4 \\
\hline \multicolumn{14}{|c|}{ Black plastic mulch } \\
\hline \multirow[t]{3}{*}{$\mathrm{N}$} & Zefyr & 204 & \pm 5 & 15.1 & \pm 0.6 & 85.3 & \pm 1.8 & 1.10 & \pm 0.05 & 11.5 & \pm 0.7 & 79.2 & \pm 1.8 \\
\hline & Honeoye & 193 & \pm 7 & 23.0 & \pm 0.7 & 63.6 & \pm 0.8 & 0.92 & \pm 0.02 & 9.8 & \pm 1.7 & 66.9 & \pm 0.4 \\
\hline & Polka & 156 & \pm 7 & 17.6 & \pm 0.3 & 65.0 & \pm 0.9 & 0.71 & \pm 0.02 & 11.7 & \pm 0.9 & 61.5 & \pm 0.7 \\
\hline \multirow[t]{3}{*}{$\mathrm{P}$} & Zefyr & 251 & \pm 6 & 14.7 & \pm 2.8 & 71.3 & \pm 3.6 & 1.01 & \pm 0.07 & 14.2 & \pm 1.0 & 89.6 & \pm 0.4 \\
\hline & Honeoye & 214 & \pm 5 & 13.9 & \pm 0.1 & 69.9 & \pm 2.6 & 0.93 & \pm 0.05 & 10.9 & \pm 1.0 & 83.7 & \pm 0.6 \\
\hline & Polka & 172 & \pm 9 & 13.3 & \pm 1.6 & 51.9 & \pm 1.9 & 0.96 & \pm 0.05 & 12.8 & \pm 0.9 & 78.4 & \pm 0.4 \\
\hline \multirow[t]{3}{*}{ A } & Zefyr & 241 & \pm 5 & 15.2 & \pm 0.7 & 76.7 & \pm 0.5 & 0.99 & \pm 0.04 & 10.6 & \pm 1.0 & 84.7 & \pm 2.2 \\
\hline & Honeoye & 221 & \pm 6 & 20.2 & \pm 0.4 & 65.1 & \pm 2.9 & 0.94 & \pm 0.05 & 9.8 & \pm 0.4 & 77.8 & \pm 2.2 \\
\hline & Polka & 204 & \pm 5 & 14.9 & \pm 0.8 & 54.7 & \pm 1.1 & 0.93 & \pm 0.04 & 11.9 & \pm 0.5 & 85.2 & \pm 0.5 \\
\hline \multicolumn{14}{|c|}{ Average } \\
\hline \multicolumn{14}{|c|}{ Mulch } \\
\hline & mulch & 212 & $\mathrm{~b}$ & 18.2 & $\mathrm{~b}$ & 66.6 & $\mathrm{a}$ & 1.03 & $\mathrm{~b}$ & 10.6 & $\mathrm{a}$ & 76.1 & $\mathrm{a}$ \\
\hline & ck plastic & 207 & $\mathrm{a}$ & 16.7 & $\mathrm{a}$ & 67.1 & $\mathrm{a}$ & 0.94 & $\mathrm{a}$ & 11.4 & $\mathrm{~b}$ & 78.6 & $\mathrm{~b}$ \\
\hline & $\mathrm{p}$ value & \multicolumn{2}{|c|}{0.000} & \multicolumn{2}{|c|}{0.000} & \multicolumn{2}{|c|}{0.209} & \multicolumn{2}{|c|}{0.000} & \multicolumn{2}{|c|}{0.001} & \multicolumn{2}{|c|}{0.000} \\
\hline \multicolumn{14}{|c|}{ Plant cover } \\
\hline $\mathrm{N}$ & & 195 & $\mathrm{a}$ & 19.3 & $\mathrm{c}$ & 70.8 & $\mathrm{c}$ & 0.99 & $\mathrm{a}$ & 10.9 & $\mathrm{a}$ & 74.6 & $\mathrm{~b}$ \\
\hline $\mathrm{P}$ & & 222 & $\mathrm{c}$ & 15.2 & $\mathrm{a}$ & 62.8 & $\mathrm{a}$ & 0.98 & $\mathrm{a}$ & 11.7 & $\mathrm{~b}$ & 83.8 & $\mathrm{c}$ \\
\hline A & & 211 & $\mathrm{~b}$ & 17.8 & $\mathrm{~b}$ & 67.0 & $\mathrm{~b}$ & 0.99 & $\mathrm{a}$ & 10.6 & $\mathrm{a}$ & 73.6 & $\mathrm{a}$ \\
\hline & $\mathrm{p}$ value & & & & & & & & & & & & \\
\hline & tivar & & & & & & & & & & & & \\
\hline & & 239 & $\mathrm{c}$ & 16.7 & $\mathrm{a}$ & 77.9 & $\mathrm{c}$ & 1.05 & $\mathrm{c}$ & 11.7 & $\mathrm{~b}$ & 82.3 & $\mathrm{c}$ \\
\hline & heoye & 215 & $\mathrm{~b}$ & 19.1 & $\mathrm{~b}$ & 64.7 & $\mathrm{~b}$ & 1.02 & $\mathrm{~b}$ & 9.8 & $\mathrm{a}$ & 75.4 & $\mathrm{~b}$ \\
\hline & & 174 & $\mathrm{a}$ & 16.6 & $\mathrm{a}$ & 58.0 & $\mathrm{a}$ & 0.90 & $\mathrm{a}$ & 11.7 & $\mathrm{~b}$ & 74.3 & $\mathrm{a}$ \\
\hline & $\mathrm{p}$ value & & & & & & & & & & & & \\
\hline
\end{tabular}

Explanations:

*Treatments: $\mathrm{N}$ - plants not covered; $\mathrm{P}$ - plants covered by polyethylene film; A - plants covered by Agronet

**Biochemical components: AN - anthocyanins; RSA - antioxidant activity as radical scavenging

Different letters in columns within each factor indicate significant differences by Duncan's multiple range test, $\mathrm{p}=0.05$ 
Table 2. Biochemical content and radical scavenging activity in strawberry fruits in 2009 ( \pm standard deviation)

\begin{tabular}{|c|c|c|c|c|c|c|c|c|c|c|c|c|c|}
\hline \multicolumn{2}{|c|}{ Treatment* } & \multicolumn{2}{|c|}{$\begin{array}{l}\text { Phenolics } \\
\mathrm{mg} \cdot 100 \mathrm{~g}^{-1}\end{array}$} & \multicolumn{2}{|c|}{$\begin{array}{c}\mathrm{AN} * * \\
\mathrm{mg} \cdot 100 \mathrm{~g}^{-1}\end{array}$} & \multicolumn{2}{|c|}{$\begin{array}{c}\text { Ascorbic acid } \\
\mathrm{mg} \cdot 100 \mathrm{~g}^{-1}\end{array}$} & \multicolumn{2}{|c|}{$\begin{array}{l}\text { Titratable } \\
\text { acidity } \%\end{array}$} & \multicolumn{2}{|c|}{$\begin{array}{c}\text { Soluble solids } \\
\text { OBrix }\end{array}$} & \multicolumn{2}{|c|}{$\mathrm{RSA}^{* * * \%}$} \\
\hline \multicolumn{14}{|c|}{ Without soil mulch } \\
\hline \multirow[t]{3}{*}{$\mathrm{N}$} & Zefyr & 265 & \pm 1 & 17.9 & \pm 0.5 & 68.6 & \pm 1.2 & 0.96 & \pm 0.04 & 9.5 & \pm 0.4 & 87.5 & \pm 1.7 \\
\hline & Honeoye & 305 & \pm 3 & 28.3 & \pm 1.7 & 80.8 & \pm 2.5 & 1.10 & \pm 0.06 & 9.4 & \pm 0.8 & 80.1 & \pm 1.5 \\
\hline & Polka & 256 & \pm 2 & 28.8 & \pm 1.1 & 64.5 & \pm 3.9 & 0.94 & \pm 0.04 & 9.8 & \pm 0.6 & 75.1 & \pm 2.7 \\
\hline \multirow[t]{3}{*}{$\mathrm{P}$} & Zefyr & 242 & \pm 1 & 17.6 & \pm 1.0 & 90.8 & \pm 1.9 & 0.82 & \pm 0.04 & 8.5 & \pm 0.8 & 81.4 & \pm 1.4 \\
\hline & Honeoye & 222 & \pm 6 & 20.0 & \pm 1.1 & 72.9 & \pm 2.9 & 0.84 & \pm 0.04 & 7.6 & \pm 0.4 & 74.4 & \pm 0.9 \\
\hline & Polka & 186 & \pm 2 & 15.0 & \pm 1.9 & 51.2 & \pm 4.4 & 0.78 & \pm 0.00 & 9.6 & \pm 0.4 & 65.9 & \pm 0.7 \\
\hline \multirow[t]{3}{*}{ A } & Zefyr & 227 & \pm 2 & 15.1 & \pm 0.6 & 83.8 & \pm 1.8 & 0.90 & \pm 0.04 & 8.9 & \pm 0.4 & 78.5 & \pm 0.5 \\
\hline & Honeoye & 196 & \pm 1 & 20.2 & \pm 0.8 & 67.6 & \pm 1.2 & 0.94 & \pm 0.06 & 8.5 & \pm 0.4 & 68.6 & \pm 1.5 \\
\hline & Polka & 172 & \pm 1 & 13.2 & \pm 0.4 & 71.0 & \pm 1.3 & 1.03 & \pm 0.04 & 10.8 & \pm 0.9 & 68.8 & \pm 1.3 \\
\hline \multicolumn{14}{|c|}{ Black plastic mulch } \\
\hline \multirow[t]{3}{*}{$\mathrm{N}$} & Zefyr & 246 & \pm 2 & 18.3 & \pm 0.9 & 72.7 & \pm 0.8 & 0.81 & \pm 0.04 & 8.9 & \pm 0.6 & 88.4 & \pm 1.0 \\
\hline & Honeoye & 245 & \pm 2 & 24.3 & \pm 1.5 & 71.9 & \pm 4.4 & 1.00 & \pm 0.06 & 9.7 & \pm 1.1 & 79.8 & \pm 0.4 \\
\hline & Polka & 226 & \pm 2 & 27.1 & \pm 0.6 & 48.7 & \pm 3.2 & 0.92 & \pm 0.12 & 9.5 & \pm 0.4 & 73.8 & \pm 1.7 \\
\hline \multirow[t]{3}{*}{$\mathrm{P}$} & Zefyr & 214 & \pm 3 & 18.2 & \pm 1.6 & 79.6 & \pm 3.3 & 0.84 & \pm 0.07 & 8.8 & \pm 1.0 & 78.6 & \pm 1.1 \\
\hline & Honeoye & 213 & \pm 1 & 16.6 & \pm 0.3 & 76.1 & \pm 1.9 & 0.76 & \pm 0.04 & 7.7 & \pm 0.7 & 75.8 & \pm 0.7 \\
\hline & Polka & 177 & \pm 2 & 14.1 & \pm 1.2 & 48.4 & \pm 2.0 & 0.82 & \pm 0.04 & 9.9 & \pm 0.6 & 66.5 & \pm 1.2 \\
\hline \multirow[t]{3}{*}{ A } & Zefyr & 252 & \pm 3 & 15.4 & \pm 0.2 & 82.1 & \pm 2.3 & 1.00 & \pm 0.06 & 8.5 & \pm 0.2 & 83.2 & \pm 0.6 \\
\hline & Honeoye & 203 & \pm 2 & 18.5 & \pm 1.1 & 74.6 & \pm 2.5 & 1.00 & \pm 0.07 & 9.3 & \pm 0.7 & 68.4 & \pm 1.5 \\
\hline & Polka & 175 & \pm 3 & 13.9 & \pm 0.6 & 54.5 & \pm 1.9 & 0.92 & \pm 0.06 & 10.3 & \pm 0.6 & 69.1 & \pm 1.4 \\
\hline \multicolumn{14}{|c|}{ Average } \\
\hline \multicolumn{14}{|c|}{ Mulch } \\
\hline \multirow{2}{*}{\multicolumn{2}{|c|}{$\begin{array}{l}\text { No mulch } \\
\text { Black plastic }\end{array}$}} & 230 & $\mathrm{~b}$ & 19.6 & $\mathrm{~b}$ & 72.4 & $\mathrm{~b}$ & 0.92 & $\mathrm{~b}$ & 9.2 & $\mathrm{a}$ & 75.6 & $\mathrm{a}$ \\
\hline & & 217 & $\mathrm{a}$ & 18.5 & $\mathrm{a}$ & 67.6 & $\mathrm{a}$ & 0.90 & $\mathrm{a}$ & 9.2 & $\mathrm{a}$ & 76.0 & $\mathrm{~b}$ \\
\hline \multicolumn{2}{|c|}{$\mathrm{p}$ value } & \multicolumn{2}{|c|}{0.000} & \multicolumn{2}{|c|}{0.000} & \multicolumn{2}{|c|}{0.000} & \multicolumn{2}{|c|}{0.000} & \multicolumn{2}{|c|}{0.972} & 0.0 & \\
\hline & at cover & & & & & & & & & & & & \\
\hline $\mathrm{N}$ & & 257 & $\mathrm{c}$ & 24.1 & $\mathrm{c}$ & 67.9 & $\mathrm{a}$ & 0.95 & $\mathrm{~b}$ & 9.5 & $\mathrm{~b}$ & 80.8 & $\mathrm{c}$ \\
\hline $\mathrm{P}$ & & 209 & $\mathrm{~b}$ & 16.9 & $\mathrm{~b}$ & 69.8 & $\mathrm{~b}$ & 0.81 & $\mathrm{a}$ & 8.7 & $\mathrm{a}$ & 73.8 & $\mathrm{~b}$ \\
\hline A & & 204 & $\mathrm{a}$ & 16.0 & $\mathrm{a}$ & 72.3 & $\mathrm{c}$ & 0.96 & $\mathrm{~b}$ & 9.4 & $\mathrm{~b}$ & 72.8 & $\mathrm{a}$ \\
\hline & $p$ value & 0. & & 0.0 & & & & 0.1 & & & & & \\
\hline & tivar & & & & & & & & & & & & \\
\hline & & 241 & $\mathrm{c}$ & 17.1 & $\mathrm{a}$ & 79.6 & $\mathrm{c}$ & 0.89 & $\mathrm{a}$ & 8.8 & $\mathrm{a}$ & 82.9 & $\mathrm{c}$ \\
\hline & leoye & 231 & $\mathrm{~b}$ & 21.3 & $\mathrm{c}$ & 74.0 & $\mathrm{~b}$ & 0.94 & $\mathrm{~b}$ & 8.7 & $\mathrm{a}$ & 74.5 & $\mathrm{~b}$ \\
\hline & & 199 & $\mathrm{a}$ & 18.7 & $\mathrm{~b}$ & 56.4 & $\mathrm{a}$ & 0.90 & $\mathrm{a}$ & 10.0 & $\mathrm{~b}$ & 69.9 & $\mathrm{a}$ \\
\hline & $p$ value & 0. & & 0.0 & & & & & & & & & \\
\hline
\end{tabular}

Explanations:

*Treatments: $\mathrm{N}$ - plants not covered; $\mathrm{P}$ - plants covered by polyethylene film; A - plants covered by Agronet

**Biochemical components: AN - anthocyanins; RSA - antioxidant activity as radical scavenging

Different letters in columns within each factor indicate significant differences by Duncan's multiple range test, $\mathrm{p}=0.05$ 
Table 3. The effect size of factors and factor interactions on biochemical contents in strawberry fruits, $\eta^{2} \%$

\begin{tabular}{|c|c|c|c|c|c|c|c|c|c|c|c|c|}
\hline \multirow{2}{*}{$\frac{\text { Factor }}{\mathrm{Y}}$} & \multicolumn{2}{|c|}{ Anthocyanins } & \multicolumn{2}{|c|}{ Phenolics } & \multicolumn{2}{|c|}{ Ascorbic acid } & \multicolumn{2}{|c|}{$\begin{array}{c}\text { Titratable } \\
\text { acidity }\end{array}$} & \multicolumn{2}{|c|}{ Soluble solids } & \multicolumn{2}{|c|}{ RSA } \\
\hline & 3.4 & $* *$ & 4.1 & $* *$ & 1.8 & $* *$ & 11.0 & $* *$ & 35.9 & $* *$ & 0.9 & $*$ \\
\hline M & 2.4 & $* *$ & 1.9 & $* *$ & 0.8 & $\mathrm{~ns}$ & 6.3 & $* *$ & 1.6 & ns & 0.8 & ns \\
\hline $\mathrm{PC}$ & 36.2 & $* *$ & 5.0 & $* *$ & 1.6 & $\mathrm{~ns}$ & 10.3 & $* *$ & 0.4 & ns & 9.3 & $* *$ \\
\hline $\mathrm{C}$ & 11.5 & $* *$ & 42.2 & $* *$ & 55.9 & $* *$ & 9.3 & $* *$ & 17.4 & $* *$ & 31.2 & $* *$ \\
\hline $\mathrm{Y} * \mathrm{M}$ & 0.1 & $\mathrm{~ns}$ & 0.3 & ns & 1.2 & $*$ & 1.6 & $* *$ & 1.7 & ns & 0.4 & ns \\
\hline $\mathrm{Y} * \mathrm{PC}$ & 10.1 & $* *$ & 24.6 & $* *$ & 3.4 & $* *$ & 7.6 & $* *$ & 6.5 & $* *$ & 17.3 & $* *$ \\
\hline $\mathrm{M} * \mathrm{PC}$ & 0.3 & $\mathrm{~ns}$ & 8.6 & $* *$ & 0.4 & $\mathrm{~ns}$ & 4.5 & $* *$ & 2.2 & $\mathrm{~ns}$ & 15.9 & $* *$ \\
\hline $\mathrm{Y}^{*} \mathrm{C}$ & 1.0 & $\mathrm{~ns}$ & 1.8 & $*$ & 3.7 & $* *$ & 7.9 & $* *$ & 5.0 & $* *$ & 1.7 & $*$ \\
\hline $\mathrm{M}^{*} \mathrm{C}$ & 0.1 & $\mathrm{~ns}$ & 0.7 & $\mathrm{~ns}$ & 2.1 & $* *$ & 2.9 & $* *$ & 0.1 & $\mathrm{~ns}$ & 0.3 & ns \\
\hline $\mathrm{PC} * \mathrm{C}$ & 16.0 & $* *$ & 1.9 & ns & 7.0 & $* *$ & 6.7 & $* *$ & 3.6 & ns & 4.3 & $* *$ \\
\hline $\mathrm{Y} * \mathrm{M} * \mathrm{PC}$ & 0.1 & $\mathrm{~ns}$ & 0.4 & $\mathrm{~ns}$ & 0.5 & $\mathrm{~ns}$ & 0.4 & ns & 0.8 & $\mathrm{~ns}$ & 12.0 & $* *$ \\
\hline $\mathrm{Y}^{*} \mathrm{M}^{*} \mathrm{C}$ & 2.7 & $* *$ & 0.6 & $\mathrm{~ns}$ & 0.6 & ns & 1.6 & $*$ & 0.2 & $\mathrm{~ns}$ & 0.1 & ns \\
\hline $\mathrm{Y} * \mathrm{PC} * \mathrm{C}$ & 6.9 & $* *$ & 3.8 & $* *$ & 5.0 & $* *$ & 6.8 & $* *$ & 1.9 & $\mathrm{~ns}$ & 1.0 & ns \\
\hline $\mathrm{M} * \mathrm{PC} * \mathrm{C}$ & 2.5 & $*$ & 0.3 & ns & 8.8 & $* *$ & 0.4 & ns & 1.5 & ns & 0.8 & ns \\
\hline $\mathrm{Y} * \mathrm{M} * \mathrm{PC} * \mathrm{C}$ & 1.4 & $\mathrm{~ns}$ & 2.3 & $*$ & 2.7 & $*$ & 6.2 & $* *$ & 0.9 & $\mathrm{~ns}$ & 1.5 & ns \\
\hline
\end{tabular}

Factors:

$\mathrm{Y}$ - year; $\mathrm{M}$ - mulch; $\mathrm{PC}$ - plant cover; $\mathrm{C}$ - cultivar

* - significant at $\mathrm{p}=0.05 ; * *$ - significant at $\mathrm{p}=0.01$; ns - not significant, by $\mathrm{F}$ test

The content of ascorbic acid in both years of the study was the highest in 'Zefyr' grown without soil mulching and with plant covering with polyethylene film in the spring. For 'Polka', the highest ascorbic acid content was found in fruits obtained from the cultivation system without soil mulching and with Agronet covering. For 'Honeoye' results differed between seasons.

The highest content of titratable acidity in 'Honeoye' was found in fruits obtained in the cultivation system where plants were grown without soil mulching and plant covering. For 'Zefyr' and 'Polka' results differed between years.

In 2008, the highest content of soluble solids in all cultivars was observed in the cultivation systems based on black plastic mulch and plant covering by polyethylene film, whereas in 2009 cultivars showed different results within growing systems.

The highest RSA in 2008 was observed in the fruits of 'Zefyr' and 'Honeoye' at the cultivation systems with black plastic mulch and plants covering with polyethylene film, whereas in 'Polka,' it was the highest in the cultivation system with black plastic mulch and Agronet covering. In 2009, in 'Honeoye' and 'Polka' fruits, the highest RSA was observed in the cultivation system without soil mulching and plant covering, whereas in 'Zefyr' it was higher where black plastic mulch was applied.

\section{CONCLUSIONS}

The strawberry biochemical content was significantly influenced by annual climatic conditions, as well as by cultivar and cultivation system. Cultivar had the greatest influence on the content of ascorbic acid, total phenolics and RSA from all studied factors. The content of anthocyanins was influenced mainly by plants covering. The annual climatic conditions had the highest impact on the content of titratable acidity and soluble solids. Soil mulching had the weakest influence on fruit phytochemical content.

\section{REFERENCES}

Del Pozo-Insfran D., Duncan C.E., Yu K.C., Talcott S.T. 2006. Polyphenolics, ascorbic acid, and soluble solids concentrations of strawberry cultivars and selections grown in a winter annual hill production system. J. Amer. Soc. Hort. Sci. 131(1): 89-96.

Diamanti J., Capocasa F., Mezzetti B., Tulipani S., Battino M. 2009. The interaction of plant genotype 
and temperature conditions at ripening stage affects strawberry nutritional quality. Acta Hort. 838: 183 186. http://www.actahort.org/books/838/838_30.htm

Giampieri F., Tulipani S., Alvarez-Suarez J.M., Quiles J.L., Mezzetti B., Battino M. 2012. The strawberry: composition, nutritional quality, and impact on human health. Nutrition. 28:9-19.

Hannum S.M. 2004. Potential impact of strawberries on human health: A review of the science. Crit. Rev. Food Sci. Nut. 44:1-17. DOI: 10.1016/j.nut.2011.08.009.

Hansawasdi C., Rithiudom S., Chaiprasart P. 2006. Quality and antioxidant activity changes during low-temperature storage of strawberry fruits. Acta Hort. 708: 301-306. http://www.actahort.org/books/708/708_51.htm

Himelrick D.G., Woods F.M., Dozier W.A. Jr, Akridge. J.R. 1996. Influence of mulch colour on strawberry production in the annual hill plasticulture system. Advances in Strawberry Research 15: 42-47.

Ikeda T., Yamazaki K., Kumakura H., Hamamoto H. 2009. Effect of high temperature on fruit quality of potgrown strawberry plants. Acta Hort. 842: 679-682. http://www.actahort.org/books/842/842_146.htm

Jaroslawska J., Juskiewicz J., Wroblewska M., Jurgonski A., Krol B., Zdunczyk Z. 2011. Polyphenol-rich strawberry pomace reduces serum and liver lipids and alters gastrointestinal metabolite formation in fructose-fed rats. Journal of Nutrition 10 (141): 1777-1783. DOI: 10.3945/jn.111.143677.

Josuttis M., Krüger E., Dietrich H. 2009. Influence of cultivation techniques on health beneficial components in strawberry. Acta Hort. 838: 205-210. http://www.actahort.org/books/838/838_34.htm

Kikas A. 1997. Strawberry investigation: the effect of different types of mulches on the formation of runner plants and berry yield. Collection of Scientific Articles of Conf. "Modern orchards: achievements and tendencies". Babtai, Lithuania. pp. 114-117.

Krüger E., Toldam-Anderson T., Dietrich H. 2009. Climatic influence on strawberry yield, quality and bioactive compounds in different European cultivation conditions. Acta Hort. 842: 903-906. http://www.actahort.org/books/842/842_199.htm

Laugale V., Lepsis J., Strautina S. 2012. Extending strawberry production season in Latvia. Acta Hort. 926: 551-557.

Manach C., Scalbert A., Morand C., Remesy C., Jimenez L. 2004. Polyphenols: food source and bioavailability. Am. J. Clin. Nutr. 79: 727-747.
Mratinić E., Milivojević J. 2009. The influence of harvest time on strawberry fruit quality (Fragaria $\times$ ananassa Duch.). Acta Hort. 842: 911-914. http://www.actahort.org/books/842/842_201.htm

Martinsson M. 1997. Strawberry growing in Sweden. Acta Hort. 439: 397-401. http://www.actahort.org/books/439/439_65.htm

Medina L. 2008. New technologies. Technological revolution. P. 175-218. In: Junta de Andalucia (Eds.), The strawberry crop in Huelva. Autonomous Vice Ministry, Spain.

Miliauskas G., Venskutonis P.R., van Beek T.A. 2004. Screening of radical scavenging activity of some medicinal and aromatic plant extracts. Food Chemistry 85: 231-237. DOI: 10.1016/j.foodchem.2003.05.007.

Moor U., Karp K., Poldma P., Pae A. 2005. Cultural systems affect content of anthocyanins and vitamin $\mathrm{C}$ in strawberry fruits. European Journal of Horticultural Science 70(4): 195-201.

Rutkowski K.P., Kruczynska D.E., Zurawicz E. 2006. Quality and shelf life of strawberry cultivars in Poland. Acta Hort. 708: 329-332. http://www.actahort.org/books/708/708_56.htm

Scalzo J., Capocasa F., Palandrani A., Mezzetti B., Battino M. 2004. Quality and nutritional value in strawberry breeding and cultivar evaluation. Acta Hort. 649: 6164. http://www.actahort.org/books/649/649_8.htm

Seglina D., Krasnova I., Ruisa S., Strautina S., Heidemane G. 2008. Research on antioxidant activity of berries grown in Latvia. Proc. of Intern. Scientific Conf. "Sustainable fruit growing: from plant to product". Latvia State Institute of Fruit- Growing, Jūrmala - Dobele, Latvia, pp. 265-273.

Singleton V.L., Orthofer R.M., Lamuela-Raventos R.M. 1999. Analysis of total phenols and other oxidation substances and antioxidants by means of Folin-Ciocalteu reagent. Methods in Enzymology 299: 152 178.

Tulipani S., Romandini S., Battino M., Bompadre S., Capocasa F., Mezzetti B. 2009. Variation in strawberry micronutrients, phytochemical and antioxidant profiles: the combined effect of genotype and storage. Acta Hort. 842: 867-872. http://www.actahort.org/books/842/842_191.htm

Wang S.Y. 2007. Antioxidant capacity and phenolic content of berry fruits as affected by genotype, preharvest conditions, maturity, and postharvest handling. pp. 147-178. In: Zhao Y. (Ed.), Berry Fruit: ValueAdded Products For Health Promotion. CRC Press, Boca Raton pp. 147-186. 
Weaver J., Briscoe T., Hou M., Goodman C., Kata S., Roos H., et al. 2009. Strawberry polyphenols are equally cytoxic to tumourigenic and normal hu- man breast and prostate lines. International Journal of Oncology 34: 777-786. DOI: 10.3892/ijo_00000203. 